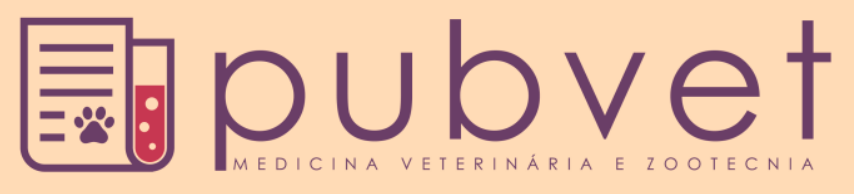

https://doi.org/10.31533/pubvet.v12n7a146.1-6

\title{
Parasitos gastrintestinais em ovinos de uma propriedade rural do estado do Piauí
}

\author{
Gilmara Ferreira Dias ${ }^{1}$, Auan Rangel Oliveira de $\operatorname{Vasconcelos}^{1} \bullet$, Tiago Paixão Ribeiro \\ de Sousa $^{1}$, Matheus Luiggi Freitas $\operatorname{Barbosa}^{1}{ }^{\bullet}$, Francisco Michael Júnior Costa ${ }^{1}{ }^{\bullet}$, \\ Jefferson Hallison Lustosa da Silva ${ }^{2}$, Felipe Pereira da Silva Barçante ${ }^{3 \bullet}$, Luanna \\ Soares de Melo Evangelista ${ }^{4 * 6}$
}

${ }^{1}$ Acadêmico(a) de Medicina Veterinária, Universidade Federal do Piauí. Teresina-PI Brasil.

${ }^{2}$ Residente em Reprodução Animal, Universidade Federal do Piauí. Teresina-PI Brasil.

${ }^{3}$ Professor Dr. do Instituto Educacional Superior Múltiplo. Timon-MA Brasil.

${ }^{4}$ Professora Dr ${ }^{a}$ do Departamento de Parasitologia e Microbiologia, Centro de Ciências da Saúde, Universidade Federal do Piauí. Teresina-PI Brasil.

*Autor para correspondência. E-mail: luannaufpi@gmail.com

RESUMO. A ovinocultura constitui uma importante atividade econômica no Nordeste do Brasil, principalmente nos estados da Bahia, Pernambuco, Ceará e Piauí. Mesmo com uma crescente demanda por seus produtos como carne e lã, a ovinocultura ainda apresenta limitações devido às condições de manejo sanitário, alimentar e reprodutivo do rebanho. $\mathrm{O}$ objetivo deste trabalho foi identificar parasitos gastrintestinais em ovinos de uma propriedade rural de Santa Cruz dos Milagres, PI, avaliados durante o período seco na região. Os animais foram escolhidos aleatoriamente, dentre os não vermifugados há mais de três meses, todas fêmeas, com idade entre 06 meses a 03 anos, totalizando 55 animais, onde foram separadas por categoria: jovens ( $\leq 12$ meses) e adultas ( $\geq 12$ meses). O trabalho foi realizado no mês de outubro de 2015. Foram colhidas amostras de fezes diretamente da ampola retal dos animais, com uso de luvas, previamente identificadas, colocadas em sacos plásticos estéreis e levadas para o Laboratório de Parasitologia do Departamento de Parasitologia e Microbiologia da Universidade Federal do Piauí, para posterior análise pela técnica de McMaster. Foi aplicado o teste do Qui-quadrado para avaliação estatística dos dados. Observou-se a presença de parasitos exemplares das famílias Strongyloididae e Trychostrongylidae, e das espécies Trichuris globulosa e Moniezia expansa em todos os animais, com diferença significativa entre a categoria animal. Estes resultados revelaram que, no período seco, os ovinos estavam bastante parasitados, inclusive, alguns animais apresentaram mais de uma parasitose associadas, com ocorrência de Haemonchus contortus nas fezes. Conclui-se que o conhecimento e o diagnóstico precoce dos parasitos em ovinos são fundamentais ao manejo sanitário do rebanho e controle de enfermidades parasitárias.

Palavras chave: diagnóstico, endoparasitos, manejo sanitário, ovinos

\section{Gastrintestinal parasites in sheep of a rural property of the state of Piauí}

ABSTRACT. Sheep farming is an important economic activity in Northeast Brazil, mainly in the states of Bahia, Pernambuco, Ceará and Piauí. Even with a growing demand for its products such as meat and wool, sheep production still presents limitations due to the conditions of sanitary, alimentary and reproductive management of the herd. The objective of this study was to identify gastrointestinal parasites in sheep of a rural property of Santa Cruz dos Milagres, PI, evaluated during the dry period in the region. The animals were 
randomly selected, among the non-wormed for more than three months, all females, aged between 06 months and 03 years, totaling 55 animals, where they were separated by category: young ( $\leq 12$ months) and adults ( $\geq 12$ months). The work was carried out in October, 2015. Fecal samples were collected directly from the rectal ampule of the animals, using gloves, previously identified, placed in sterile plastic bags and taken to the Laboratory of Parasitology of the Department of Parasitology and Microbiology of Federal University of Piauí, for later analysis by the McMaster technique. The Chi-square test was applied for statistical evaluation of the data. It was observed the presence of exemplary parasites of the families Strongyloididae and Trychostrongylidae, and of the species Trichuris globulosa and Moniezia expansa in all the animals, with significant difference between the animal category. These results revealed that, in the dry period, sheep were highly parasitized, and some animals had more than one associated parasitosis, with Haemonchus contortus occurring in the faeces. It is concluded that the knowledge and the early diagnosis of the parasites in sheep are fundamental to the sanitary management of the herd and control of parasitic diseases.

Keywords: diagnosis, endoparasites, sanitary management, sheep

\section{Parásitos gastrointestinales en ovinos de una propiedad rural del estado de Piauí}

RESUMEN. La ovinocultura constituye una importante actividad económica en el Nordeste de Brasil, principalmente en los estados de Bahía, Pernambuco, Ceará y Piauí. Incluso con una creciente demanda por sus productos como carne y lana, la ganadería ovina todavía presenta limitaciones debido a las condiciones de manejo sanitario, alimentario y reproductivo del rebaño. El objetivo de este trabajo fue identificar parásitos gastrointestinales en ovinos de una propiedad rural de Santa Cruz de los Milagres, PI, evaluados durante el período seco en la región. Los animales fueron elegidos aleatoriamente, entre los no vermifugados hace más de tres meses, todas las hembras, con edad entre 6 meses a 3 años, totalizando 55 animales, donde fueron separadas por categoría: jóvenes ( $\leq 12$ meses) y adultas ( $\geq 12$ meses). El trabajo se realizó en el mes de octubre de 2015. Se tomaron muestras de heces directamente de la ampolla rectal de los animales, con el uso de guantes previamente identificados, colocados en bolsas plásticas estériles y llevadas al Laboratorio de Parasitología del Departamento de Parasitología y Microbiología Universidad Federal de Piauí, para posterior análisis por la técnica de McMaster. Se aplicó la prueba del Chi-cuadrado para la evaluación estadística de los datos. Se observó la presencia de parásitos ejemplares de las familias Strongyloididae y Trychostrongylidae, y de las especies Trichuris globulosa y Moniezia expansa en todos los animales, con diferencia significativa entre la categoría animal. Estos resultados revelaron que, en el período seco, los ovinos estaban bastante parasitados, incluso, algunos animales presentaron más de una parasitosis asociada, con ocurrencia de Haemonchus contortus en las heces. Se concluye que el conocimiento y el diagnóstico precoz de los parásitos en ovinos son fundamentales para el manejo sanitario del rebaño y control de enfermedades parasitarias.

Palabras clave: diagnóstico, endoparasitos, manejo sanitario, ovinos

\section{Introdução}

A ovinocultura vem ganhando destaque no agronegócio brasileiro. Em 2016, o IBGE (2016) registrou mais de 18 milhões de ovinos no Brasil, dos quais mais da metade (63\%) concentra-se na região nordeste do país. O Piauí se manteve em $4^{\circ}$ lugar do Nordeste como produtor de ovinos, tanto de criação artesanal como comercial, ficando atrás apenas dos estados da Bahia, Pernambuco e Ceará. Carne e lã estão entre os principais produtos produzidos por estes animais (MAPA, 2015).

Apesar de a ovinocultura ser uma atividade econômica de grande importância, principalmente para o nordeste, no decorrer dos anos vem encontrando dificuldades para seu desenvolvimento e as parasitoses gastrintestinais 
constituem o principal fator limitante para a produção de ovinos em todo o mundo (Vieira, 2003; Moleto et al., 2013).

Entre os endoparasitos gastrintestinais de ovinos, os mais frequentemente encontrados fazem parte da família Trichostrongylidae (Vieira, 2003). Nessa família, seus principais representantes são: Haemonchus contortus e Trichostrongylys axei, que parasitam o abomaso; e Trichostrongylus colubriformis e Cooperia sp., que habitam o intestino delgado; além de espécies de outras famílias que também parasitam esses animais, como Strongyloides papillosus e Bunostomum trigonocephalum em intestino delgado e Oesophagostomum columbianum, Trichuris ovis e Trichuris globulosa, endoparasitos de intestino grosso (Andriola et al., 2011; Costa et al., 2011; Fonseca et al., 2013). Estes são responsáveis por diminuir o desenvolvimento dos animais, principalmente dos mais jovens, além de aumentar a mortalidade (Yoshihara, 2012).

O principal parasito gastrintestinal de ovinos é o Haemonchus contortus, que tem alto grau de patogenicidade, acarretando sérios prejuízos econômicos na ovinocultura mundial (Amarante, 2005; Afonso et al., 2013), além disso, esse helminto parasita animais de todas as faixas etárias e compromete não só o desenvolvimento corporal, como também interfere na qualidade de seus produtos e sub-produtos (Endo et al., 2014).

Há vários fatores que influenciam a dinâmica populacional de helmintos num rebanho, principalmente relacionados às condições climáticas de cada região (Fonseca et al. 2013), pois podem interferir diretamente sobre as formas evolutivas desses parasitos, dentro e fora de seus hospedeiros.

Dada a importância do diagnóstico precoce das parasitoses na ovinocultura, objetivou-se com este trabalho, identificar os parasitos gastrintestinais em ovinos de uma propriedade rural do município de Santa Cruz dos Milagres, Piauí, avaliados durante o período seco na região.

\section{Material e Métodos}

O experimento foi realizado na Fazenda Nova Vista, município de Santa Cruz dos Milagres, na região centro-sul do estado do Piauí, localizado a uma latitude $05^{\circ} 48^{\prime} 01^{\prime \prime}$ sul e uma longitude $41^{\circ} 57^{\prime} 34^{\prime \prime}$ oeste, estando a uma altitude de 160 metros e distante aproximadamente $200 \mathrm{~km}$ da capital piauiense.
Os ovinos foram escolhidos aleatoriamente, dentre os não vermifugados há mais de três meses, com idade entre seis meses a três anos, todos do sexo feminino, totalizando 55 animais. Elas foram separadas por categoria: fêmeas jovens $(\leq 12$ meses) e adultas ( $\geq 12$ meses), mestiças de Dorper com Santa Inês e mantidas sob criação semiextensiva. O trabalho foi realizado durante o mês de outubro de 2015, considerado período seco na região.

Foram colhidas amostras de fezes desses animais diretamente da ampola retal, com o uso de luvas, sendo previamente identificadas em sacos plásticos estéreis, colocadas em isopor com gelo reciclável e levadas para o Laboratório de Parasitologia do Departamento de Parasitologia e Microbiologia do Centro de Ciências da Saúde da Universidade Federal do Piauí (DPM/CCS/UFPI), para posterior análise.

As amostras foram avaliadas quanto ao aspecto macroscópico, colocadas em meio conservante com $1.5 \mathrm{~g}$ de acetato de sódio, $2.9 \mathrm{~mL}$ de ácido acético e $4 \mathrm{~mL}$ de formaldeído (SAF) em 92,5 mL de água destilada (Neves, 2012) e preservadas sob refrigeração $\left(4^{\circ} \mathrm{C}\right)$ até a realização dos exames parasitológicos de fezes.

As análises laboratoriais foram determinadas pela técnica de McMaster, descrita por Gordon \& Whitlock (1939), por contagem de ovos por grama de fezes (OPG). Após o procedimento da técnica e com auxílio da câmara de McMaster, as amostras foram avaliadas por microscopia óptica em objetivas de 10x e 40x, para a confirmação da infecção parasitária.

\section{Resultados e Discussão}

A maioria dos animais deste trabalho pertencia a categoria fêmeas maiores de 12 meses de idade $(89.1 \%)$, sendo consideradas adultas. A Tabela 1 mostra a classificação por idade dos animais objeto deste estudo.

Tabela 1. Idade e sexo dos ovinos avaliados durante o período do experimento (n/\%).

\begin{tabular}{llll}
\hline Animais & \multicolumn{2}{c}{ Idade } & Total \\
& $(\leq 12$ meses $)$ & $(\geq 12$ meses $)$ & \\
\hline Fêmeas & $06(10,9 \%)^{\mathrm{a}}$ & $49(89,1 \%)^{\mathrm{b}}$ & $55(100 \%)$ \\
\hline${ }^{1}$ Letras diferentes na mesma linha representam diferença \\
significativa entre os grupos pelo teste do Qui-quadrado \\
$(\mathrm{P}<0,05)$.
\end{tabular}


Durante o experimento, considerado o período seco no estado do Piauí, foi diagnosticada a presença de parasitos gastrintestinais nos ovinos avaliados, sendo os principais exemplares encontrados pertencentes às famílias Strongyloididae e Trychostrongylidae, também denominados STA, bem como helmintos das espécies Trichuris globulosa e Moniezia expansa, conforme descritos na Tabela 2.

Tabela 2. Parasitos encontrados nos ovinos avaliados durante o experimento, conforme a categoria animal $(\mathrm{n} / \%)$

\begin{tabular}{lcc}
\hline \multirow{2}{*}{ Endoparasitas } & \multicolumn{2}{c}{ Animais (\%) } \\
\cline { 2 - 3 } & $\mathrm{F}(\leq 12 \mathrm{meses})$ & $\mathrm{F}(\geq 12 \mathrm{meses})$ \\
\hline STA & $04(07,3 \%)^{\mathrm{aA}}$ & $42(76,4 \%)^{\mathrm{bA}}$ \\
Trichuris sp. & $00(00,0 \%)^{\mathrm{aB}}$ & $05(09,1 \%)^{\mathrm{bB}}$ \\
Moniezia expansa & $02(03,6 \%)^{\mathrm{aAB}}$ & $02(03,6 \%)^{\mathrm{aB}}$ \\
\hline
\end{tabular}

Legenda: F - Fêmeas. ${ }^{1}$ Letras minúsculas diferentes na mesma linha representam diferença significativa entre os grupos pelo teste do Qui-quadrado $(\mathrm{P}<0,05) .{ }^{2}$ Letras maiúsculas diferentes na mesma coluna representam diferença significativa dentro dos grupos pelo teste do Quiquadrado $(\mathrm{P}<0,05)$.

Os resultados revelaram que os animais maiores de 01 ano estavam bastante parasitados, representando uma diferença significativa entre o grupo mais jovem, inclusive, em alguns deles foi possível observar mais de uma parasitose associada. Essa informação pode ser justificada pelo número de animais ser maior nessa categoria animal.

Diarreia foi o principal sinal clínico observado na maioria das ovelhas, sendo que também foram identificados vermes adultos de Haemonchus contortus nas fezes, conforme pode ser visualizado na Figura 1. Fato este, possivelmente, relacionado ao sistema de criação empregado na fazenda ou ao baixo manejo sanitário utilizado. Outro fator pode ser dos animais não estarem vermifugados à época da realização dos exames, podendo ter servido de fonte de infecção para os demais ovinos do rebanho, inclusive para os mais jovens. Vale ressaltar que a época do ano favoreceu o aumento da incidência de parasitos nos animais.

O parasitismo por nematódeos gastrintestinais sofre influência direta da precipitação das chuvas e embora a região nordeste do Brasil seja afetada pela seca, influenciando na viabilidade de larvas no meio ambiente, estas podem sofrer adaptações no hospedeiro e continuar seu parasitismo, muitas vezes em hipobiose, e a temperatura e a umidade são os principais fatores ambientais que condicionam o desenvolvimento, a sobrevivência e a transmissão dos estágios de vida livre aos seus hospedeiros, interferindo na elevada carga parasitária em ovinos (Ahid et al., 2008; Fonseca et al., 2013).

Nematóides da família Trichostrongylidae estão frequentemente relacionados às infecções parasitárias em rebanhos ovinos e os gêneros de maior importância dessa família são o Haemonchus sp. e o Trichostrongylus sp. (Andriola et al., 2011).

Por conta do hematofagismo do Haemonchus contortus, ele pode causar anemia severa, manifestada por sintomatologia de palidez das mucosas e edema da região submandibular, podendo levar a morte de animais portadores com elevada carga parasitária (Amarante, 2005; Monteiro, 2014), sinais clínicos observados em alguns animais deste rebanho.

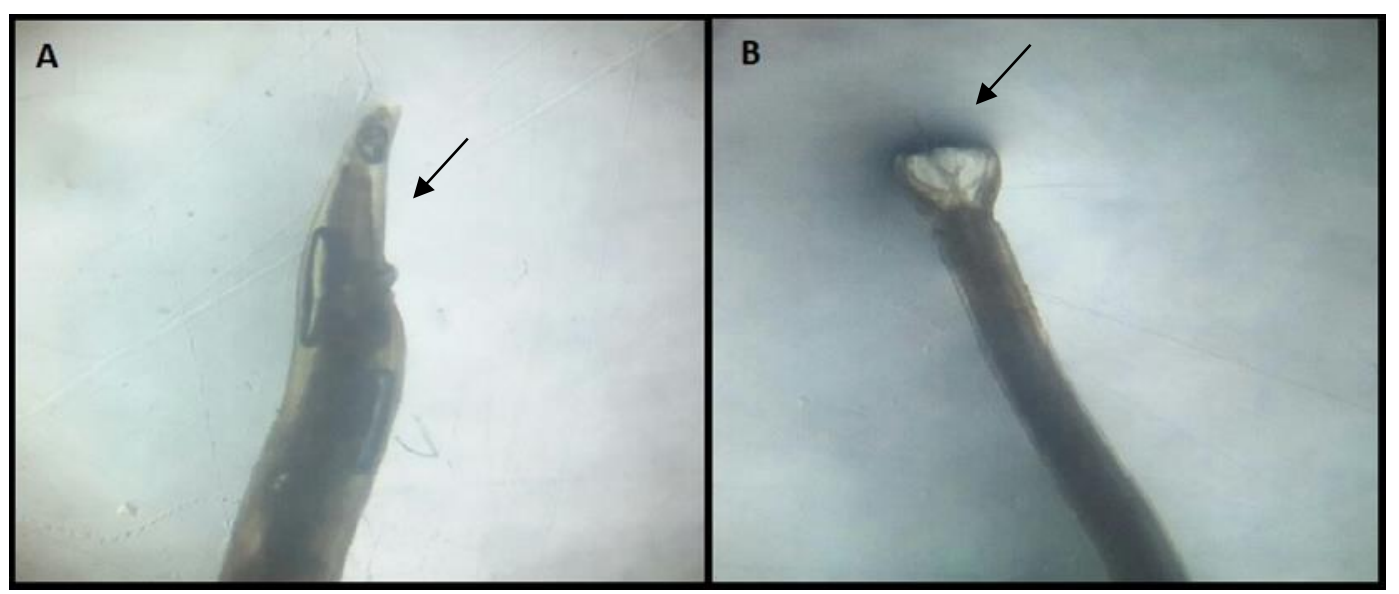

Figura 1. Adultos de Haemonchus contortus. A. Região posterior da fêmea (observar processo vulvar); B. Região posterior do macho (observar raio dorsal em forma de "Y"). 
Quando adultos, estes vermes apresentam papilas cervicais e uma lanceta no interior da cápsula bucal. Os adultos machos apresentam espículos em ganchos e raio dorsal pequeno em forma de " $Y$ " e as fềmeas apresentam três tipos de processos na região vulvar, sendo o linguiforme (grande e proeminente) o mais fácil de ser observado (Endo et al., 2014), como visualizado na figura supracitada.

Apesar de frequentemente encontrados, a literatura mostra poucos resultados de parasitos em ovinos nos rebanhos do Piauí. Em um trabalho realizado em Valença, também no estado do PI, foi observada a presença de Haemonchus contortus como o helminto gastrintestinal mais prevalente nos rebanhos caprinos avaliados (Girão et al., 1980).

O gênero Strongyloides é o principal representante da família Strongyloididae. A migração das larvas pertencentes a esse gênero pelos pulmões causa perturbações broncopulmonares, que podem desencadear em processos inflamatórios como bronquite e pneumonia. As perturbações gastrintestinais são bem evidentes, comuns e graves, e a espécie Strongyloides stercoralis tem caráter zoonótico (Monteiro, 2014).

Ovos de Trichuris sp. foram encontrados em cinco fêmeas adultas desta pesquisa, representando $9.1 \%$ do rebanho avaliado. Outros trabalhos também têm relacionado este parasito em seus resultados (Ahid et al., 2008), inclusive o Trichuris globulosa teve seu pico de incidência também na época seca em trabalho realizado no Rio Grande do Norte (Fonseca et al. 2013).

Proglotes de Moniezia expansa foram observadas em quatro animais, sendo duas jovens e duas adultas (7.2\%), sem diferença estatística entre os grupos, representando um resultado superior ao encontrado por Ahid et al. (2008) em caprinos $(4.7 \%)$ e em ovinos (4.8\%) e, inferior ao observado por Martins Filho \& Menezes (2001), em que $11.8 \%$ das amostras apresentaram ovos de Moniezia sp.

A associação parasitária de nematódeos e cestódeos em caprinos e ovinos é relativamente comum e os sinais clínicos, geralmente, ficam exacerbados nos animais. Conhecer os gêneros e/ou espécies de parasitos que ocorrem no rebanho pode contribuir para a elaboração de medidas de prevenção e controle dessas parasitoses (Martins Filho \& Menezes, 2001).
Almeida et al. (2005) descreveram, ainda, que a integridade dos bolos fecais pode torná-los potenciais reservatórios de larvas, favorecendo a sobrevivência prolongada desses estágios de vida livre no meio ambiente, aumentando o risco de infecção aos animais do rebanho.

\section{Conclusão}

O parasitismo por nematódeos e cestódeos gastrintestinais é existente em ovinos da região de Santa Cruz dos Milagres, Piauí, durante o período seco. O conhecimento e o diagnóstico destes parasitos na ovinocultura são indispensáveis para o manejo adequado e o controle de enfermidades parasitárias, uma vez que refletem um grande impacto sanitário e econômico para a produção animal.

\section{Referências Bibliográficas}

Afonso, V. A. C., Costa, R. L. D., Soares Filho, C. V., Cunha, E. A., Perri, S. H. V. \& Bonello, F. L. 2013. Supplementation with protected fat to manage gastro-intestinal nematode infections in Santa Ines sheep. Semina: Ciências Agrárias, 34(3), 1227-1238.

Ahid, S. M. M., Suassuna, A. C. D., Maia, M. B., Costa, V. M. M. \& Soares, H. S. 2008. Parasitos gastrintestinais em caprinos e ovinos da região oeste do Rio Grande do Norte, Brasil. Ciência Animal Brasileira, 9(1), 212-218.

Almeida, L. R., Castro, A. A., Silva, F. J. M. \& Fonseca, A. H. 2005. Desenvolvimento, sobrevivência e distribuição de larvas infectantes de nematóides gastrintestinais de ruminantes, na estação seca da baixada fluminense, RJ. Revista Brasileira de Parasitologia Veterinária, 14(3), 89-94.

Amarante, A. F. T. 2005. Controle de verminose ovina. Revista do Conselho Federal de Medicina Veterinária, 34, 19-30.

Andriola, L. C., Oliveira, S. V. F. B., Pereira, J. S., Coelho, W. A. C. \& Ahid, S. M. M. 2011. Diagnóstico de principais helmintos em caprinos no município de Grossos-RN. Revista Científica de Produção Animal, 13(1), 141144.

Costa, V. M. M., Simões, S. V. D. \& Riet-Correa, F. 2011. Controle das parasitoses gastrintestinais em ovinos e caprinos na região semiárida do Nordeste do Brasil. Pesquisa Veterinária Brasileira, 31(1), 65-71.

Endo, V. T., Oliveira, T. C., Cabral, A. P. M., Sakamoto, C. A. M., Ferraro, G. C., Pereira, V., 
Mazzucatto, B. C. 2014. Prevalência dos helmintos Haemonchus contortus e Oesophagostomum columbianum em pequenos ruminantes atendidos no setor de Anatomia Patológica - UEM. Revista de Ciência Veterinária e Saúde Pública, 1(2), 112-118.

Fonseca, Z. A. A. S., Pereira, J. S., Bezerra, A. C. A., Avelino, D. B., Marques, A. S. C., Paiva, K. A. R., ... Ahid, S. M. M. 2013. Helmintos gastrintestinais de caprinos leiteiros do Município de Afonso Bezerra, Rio Grande do Norte, Brasil. PUBVET, 7(19), Ed. 242, 1598.

Girão, E. S., Girão, R. N. \& Medeiros, L. P. 1980. Prevalência e variação estacional de helmintos gastrintestinais de caprinos no município de Valença do Piauí (Circular Técnica). Embrapa, $\mathrm{n}^{\circ} 01$, mês 09 .

Gordon, H. M. \& Whitlock, H. V. 1939. A new technique for counting nematode eggs in sheep faeces. Journal of the Council for Scientific and Industrial Research, 12(1), 50-52.

IBGE, Instituto Brasileiro de Geografia e Estatística. 2016. Produção da Pecuária Municipal, 44, 1-53. Disponível em: $<$ https://biblioteca.ibge.gov.br/visualizacao/pe riodicos/84/ppm_2016_v44_br.pdf $>$. Acesso em: 05 de Fevereiro de 2018

MAPA. 2015. Informativo Caprinos e Ovinos. Disponível em: $<$ http://www.agricultura.gov.br/animal/especi es/caprinos-e-ovinos>. Acesso em: $05 \mathrm{de}$ Fevereiro de 2018.

Martins Filho, E. \& Menezes, R. C. A. A. 2001. Parasitos gastrintestinais em caprinos (Capra hircus) de uma criação extensiva na microrregião de Curimataú, Estado da Paraíba, Brasil. Revista Brasileira de Parasitologia Veterinária, 10, 41-44.

Moleto, M. B., Veríssimo, C. J., Amarante, A. T., Wyk, J. A. V., Chagas, A. C. S., Araújo, J. V. \& Borges, F. A. 2013. Alternativas para o controle de nematoides gastrintestinais de pequenos ruminantes. Arquivos do Instituto Biológico, 80(2), 253-263.

Monteiro, S. G. 2014. Parasitologia na Medicina Veterinária (1a ed., 356 p.). Roca, São Paulo, BR.

Neves, D. P. 2012. Parasitologia Humana (12a ed., 546 p.). Atheneu, Rio de Janeiro, BR.

Vieira, L. S. 2003. Alternativas de controle da verminose gastrintestinal dos pequenos ruminantes (Circular técnica 29, 10 p.). Sobral, Ceará, Ministério da Agricultura, Pecuária e Abastecimento.

Yoshihara, E. 2012. Avaliação de métodos alternativos no controle de nematódeos gastrintestinais em ovinos. Pesquisa \& Tecnologia, 9(2), 1-5.

Recebido: 5 Mai. 2018.

Aprovado: 23 Mai.. 2018.

Publicado: 23 Jul. 2018.

Licenciamento: Este artigo é publicado na modalidade Acesso Aberto sob a licença Creative Commons Atribuição 4.0 (CC-BY 4.0), a qual permite uso irrestrito, distribuição, reprodução em qualquer meio, desde que o autor e a fonte sejam devidamente creditados. 